\title{
Design and Realization of the Monomer Battery Monitor Module in the BMS
}

\author{
Zhu Ya-qi ${ }^{1, a}$,Li Jun-qiu ${ }^{2, \text { b }}$,Fan Guang-chong ${ }^{3, \text { c }}$ \\ ${ }^{1,2,3}$ School of Mechanical and Vehicular Engineering , Beijing Institute of Technology , Beijing \\ 100081,P.R.China \\ ayaqigg@163.com,'lijunqiu@bit.edu.cn,'fanguangchong@126.com
}

Keywords: Battery monitor, BMS, LTC6802

\begin{abstract}
This paper presents a monomer battery monitor module in the BMS. It can collect the battery monomer voltage and temperature precisely and take appropriate measures according to different cases. It uploads the necessary data to the main controller of the BMS through the CAN bus. The realization of this module is based on the microcontroller MC9S12DP256 and the multicell battery monitoring integrate circuits LTC6802. The hardware design and the software design for serial peripheral interface (SPI) communication are given. Moreover, a thermal model of a monomer battery is built to decide the specific spots to place the thermistors or temperature sensors. The off-line test shows that the module is compact in structure, accurate and meet the requirements of stabilization and reliability.
\end{abstract}

\section{Introduction}

Electric vehicles use the on-board power batteries as their power sources. The most commonly used batteries in electric vehicles are the lithium-ion batteries. Compared to $\mathrm{NiMH}$ and other batteries, lithium-ion batteries have lower self-discharge rate, higher cell voltage and superior energy density which has make it become the first choice for the power battery of electric vehicle(EV)[1,2]. However, the voltage in each cell has to be within the range of $2.5 \mathrm{~V}$ to $4.25 \mathrm{~V}$, otherwise the lithium-ion battery would be driven into overcharge or over-discharge, which may respectively result in the swell and explosion of the cell or a shorter battery life and a lower battery capacity. Also, if the battery temperature is beyond a reasonable range, the batteries may catch fire or show a bad performance. Therefore, it is significant to include a monomer battery monitor module(MBMM) in a battery management system (BMS) to monitor the cell voltage and temperature[3,4].

\section{The architecture of the monomer battery monitor module (MBMM)}

The main functions of the monomer battery monitor module(MBMM) include: real-time measurement of the battery monomer voltage and temperature, informing the presser immediately when there are overcharged or over-discharged batteries, passive or active equalization for overcharged batteries, control of the cooling system according to the batteries temperature and communicating with the processor of BMU through the CAN bus[5].

Based on the demands mentioned above, this paper developed a monomer battery monitor module (MBMM). The module uses MC9S12DP256 chip as the controller which processes the battery voltage and temperature data and it uses LTC6802 from linear company as the multicell battery stack monitor. The MC9S12DP256 microcontroller unit is a 16-bit device composed of standard on-chip peripherals including a 16-bit central processing unit, 256K bytes of Flash EEPROM, 12K bytes of RAM, 4K bytes of EEPROM, three serial peripheral interfaces (SPI) and five CAN 2.0 A, B software compatible modules (MSCAN12). The microcontroller communicates with the controller of the BMS reliably through the CAN bus. The LTC6802 is a complete battery monitoring IC that includes a 12-bit ADC, a precision voltage reference, a high voltage input multiplexer and a serial interface. Each LTC6802 can measure up to 12 series connected battery cells with an input common mode voltage up to $60 \mathrm{~V}$. Using a unique level-shifting serial interface, 
multiple LTC6802 devices can be connected in series, without optocouplers or isolators, allowing for monitoring of every cell in a long string of series-connected batteries. When multiple LTC6802 devices are connected in series they can operate simultaneously, permitting all cell voltages in the stack to be measured within 13ms. Each cell input has an associated MOSFET switch for discharging overcharged cells. The LTC6802 also has two thermistor inputs and an on-board temperature sensor, which can monitor the battery temperature and the chip temperature respectively.

The monomer battery monitor module block diagram is shown in figure 1 . The MC9S12DP256 monitors three battery stacks through three SPIs. Each of the three battery stacks contains $\mathrm{M}$ LTC6802 devices while each LTC6802 monitors 12 battery cells' voltage and temperature. It means every microcontroller can manage up to $36^{*} \mathrm{M}$ cells, which are usually enough for an electric vehicle. The battery stack is shown in figure 2. As can be seen from it, the MC9S12DP256 only communicates with the bottom LTC6802 while the command can be sent to each one by the daisy-chained bus. And also, the data can be sent back from each LTC6802 device to the microcontroller.

LTC6802 can be used as a simple monitoring circuit with no serial interface. When in this mode, the interrupt status is indicated on the SDO pin using the toggle or level polling mode. Unlike serial port polling commands, however, the toggling is independent of the state of the CSBI pin. It is quite practical in the battery monitor module because an interruption happens only when there are cells overvoltage or undervoltage. The microcontroller can have enough spare time to monitor the temperature or communicate with the main controller of the BMU.

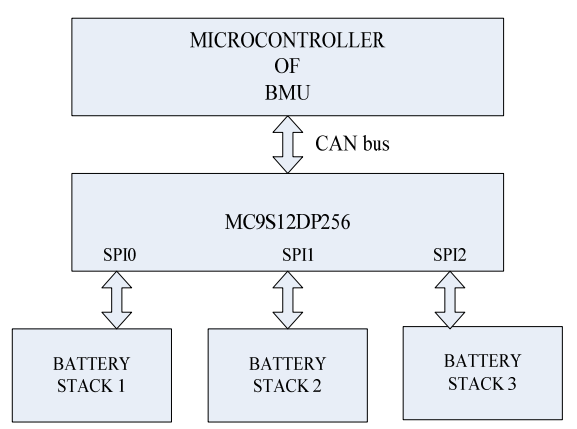

Fig.1 Structure of MBMM

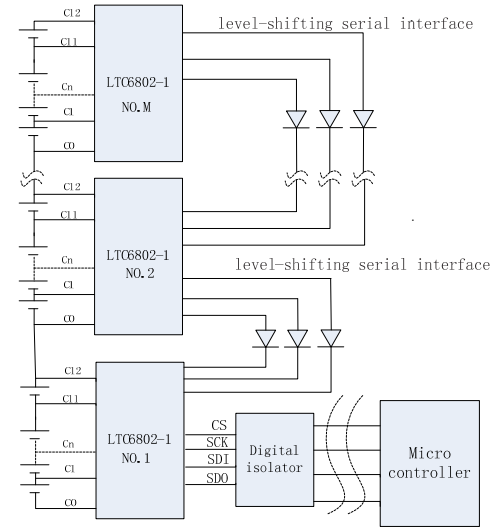

Fig.2 Structure of a battery stack

Figure 3 shows the program flow chart in this mode. After the initialization of LTC6802, The MC9S12DP256 sends the over-voltage and under-voltage threshold to LTC6802 through SPI. After the polling mode and the UV/OV comparator period are set, the cell voltage A/D conversions are executed once per 13ms by LTC6802 automatically. If none of the cells is over-voltage or under-voltage, SDO line is high and no interrupt happens. If SDO line is low, which means there are cells in over-voltage or under-voltage, the host processor will detect an interrupt and execute the battery equalization or other program.

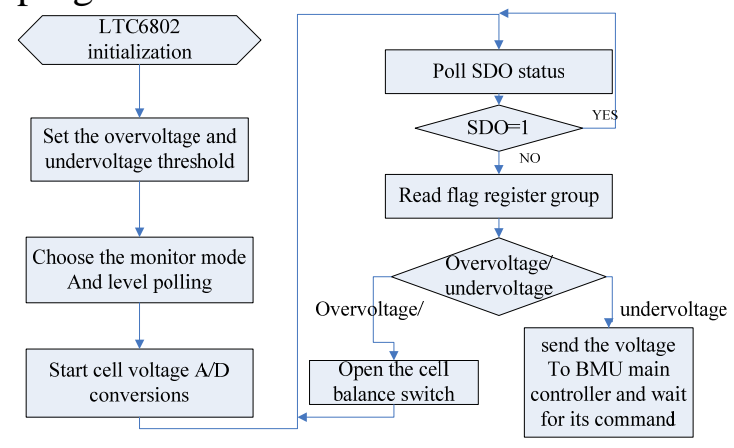

Fig.3 LTC6802 program flow in a monitor mode

\section{The equalization management of the battery cells}




\section{equalization management strategy}

Equalization management is based on the inconsistency analysis of the cells. Battery cells inconsistency is usually evaluated by the output voltage of a cell. Intuitive as it is, it can not show the inside difference between the battery cells effectively. The output voltage is influenced by the internal resistance as well as the polarizing voltage. The former influence can be neglected when the current is zero and the latter influence can be eliminated by adequate cell standing.

For battery cells used in a pure electric vehicle, the consistency is evaluated when charging is finished. Set a voltage threshold and measure the voltage of each cell. Typical cases are shown in figure 4. The voltage threshold of inconsistency estimation is U. For case 1, the voltage of three battery stacks are all below the threshold, which means there is no chance for over charge next time. Thus there is no need to equalize. For case 2, the voltage of some cells in battery stack 2 are above the threshold, which should be picked out and be equalized seperately. Stack 1 and stack 3 do not need to be equalized. For case 3, all cells voltage in battery stack 1 is above the threshold while only some cells voltage in battery stack 2 is above the threshold, so all cells in stack 1 and the over-voltaged cells in stack 2 should be equalized. For case 4, the voltage of all cells in battery stack 4 are above the threshold. None of them needs to be equalized.

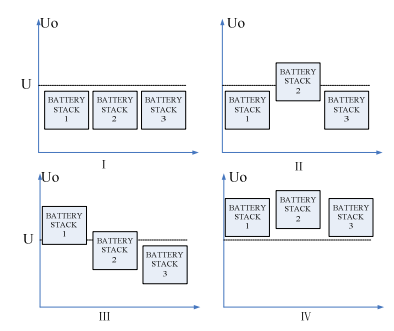

Fig. 4 Equalization management reference

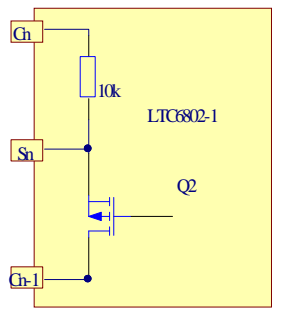

Fig. 5 Internal balance circuit of LTC6802

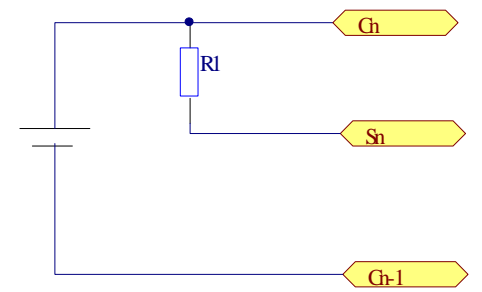

Fig. 6 External equalization circuit using the internal MOSFETs

\section{Equalization realization by LTC6802}

The LTC6802 also contains a circuitry to balance cell voltages. Each S pin output has an internal $\mathrm{N}$-channel MOSFET for discharging. The internal circuit is shown in block diagram figure 5. It provides two approaches to balance battery cells and both of them are passive equalization. Internal MOSFETs can be used to discharge cells directly. As is shown in figure 6, an external resistor should be connected in series with the NMOS to dissipate heat outside of the LTC6802 package. When using the internal MOSFETs to discharge cells, the die temperature should be monitored. These internal MOSFETs can also be used to control external balancing circuits. Figure 7 shows the S pin controlling an external balancing circuit. The LTC6802 makes no decisions about turning on/off the internal MOSFETs. This is completely controlled by the host processor. The host processor writes values to a register inside the LTC6802 to control the switches. Figure 8 illustrates the practical circuit to balance the battery, which has been proved to balance very effectively.

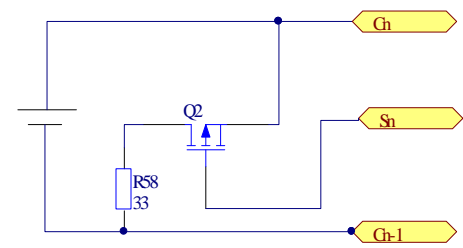

Fig.7 external balancing circuit

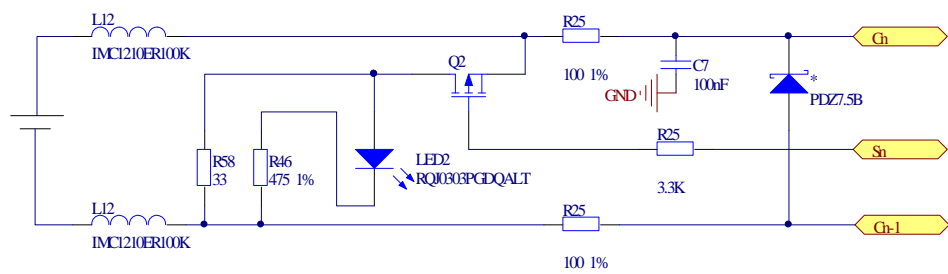

Fig.8 Practical circuit for external balance

\section{The thermal management of the batteries}

Thermal management is an important part of the monomer battery monitor module. The main tasks of thermal management are heat radiation and heating. In most cases, the temperature of batteries will rise along with discharging due to the internal resistance power consumption. So heat radiation is the main task for the module here. Temperature sensors or thermistors are placed on crucial spots of the battery surface. Therefore, it is important to know the temperature distribution 
of a cell. In this paper, a thermal model of a monomer battery is built in ANSYS software[6]. The initial condition is

$$
T(x, y, z, 0)=T_{0}
$$

$T_{0}$ is the initial temperature.

The basic formulas of convective heat transfer is given by Newton cooling formula,

$$
\begin{array}{r}
\quad-\lambda_{x} \frac{\partial T}{\partial x}=\alpha_{x}\left|T_{w}-T_{e}\right|, \quad x=0 \text { and } l \\
-\lambda_{y} \frac{\partial T}{\partial y}=\alpha_{y}\left|T_{w}-T_{e}\right|, \quad y=0 \text { and } b \\
-\lambda_{z} \frac{\partial T}{\partial z}=\alpha_{z}\left|T_{w}-T_{e}\right|, \quad z=0 \text { and } h
\end{array}
$$

In formulas above, $\alpha_{x}, \alpha_{y}, \alpha_{z}$ are the heat transfer coefficients between cell surface and the ambiance. $T_{w}$ is the surface temperature of a battery. $T_{e}$ is the environmental temperature. $l, b$ and $h$ are the length, thickness and height of the battery respectively.

Commonly, the heat transfer coefficient is $5 \mathrm{~W} /\left(\mathrm{m}^{2} \cdot \mathrm{K}\right)$ under the natural convection condition while it is $10 \mathrm{~W} /\left(\mathrm{m}^{2} \cdot \mathrm{K}\right)$ under forced air cooling condition. The simulation model adopts the air cooling condition.

The model accuracy and calculation time is influenced by the time step. The initial time step is estimated by

$$
\text { ITS }=\frac{\delta^{2}}{4 \alpha}
$$

In the formula above, $\delta$ is the unit length where the heat gradient is the biggest in the heat current direction. $\alpha$ is the temperature transfer coefficient, which is equal to the heat transfer coefficient divided by the product of the density and specific heat. When discharge the battery in 1C, the time step is set to 60s and 20s separately.

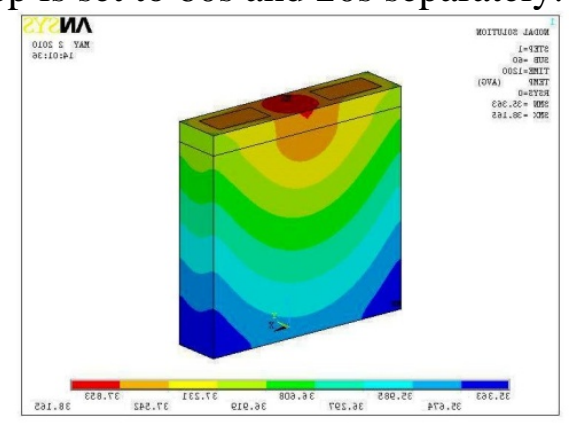

Fig.9 temperature distribution of a square battery cell

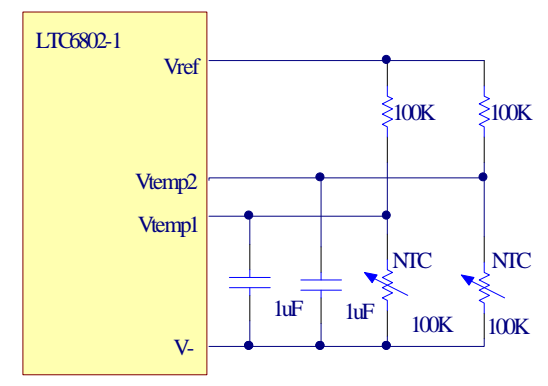

Fig.10 the circuit of temperature measurement

Figure 9 shows the temperature distribution of a square battery cell under $1 \mathrm{C}$ discharge in $25^{\circ} \mathrm{C}$. The hot spot concentrates on the top of a cell, which means most of the temperature sensors or thermistors can be placed on the top of a battery stack instead of between cells inside, where the sensors and wires are hard to deposit.

The realization of thermal management in this module is mainly executed by LTC6802 with the temperature measurement function and fans drived by relays. Figure 10 shows the circuit of the temperature measurement. NTC stands for negative temperature coefficient. Receiving a temperature measurement command, the LTC6802 will start AD conversions of the Vtemp ports input and record the results in the registers, which can be read by the host processor later. The temperature can be acquired in the resistance-temperature table of the NTC. For accurate temperature control, more temperature sensors or thermistors should be placed inside or on the surface of the battery stack, where a multiplexer may be needed. 


\section{Accuracy analysis}

The measurement accuracy of the monomer battery monitor module can be evaluated by comparing the LTC6802 measurement results and the true voltage. The experimental subject is a lithium-ion battery with a capacity of 35Ah and a voltage rating of 3.7V. Figure 11 shows the voltage curve when the battery is discharged in 4C. As is shown in the figure 11 . These two curves almost coincide with each other, which shows a perfect accuracy of the module.

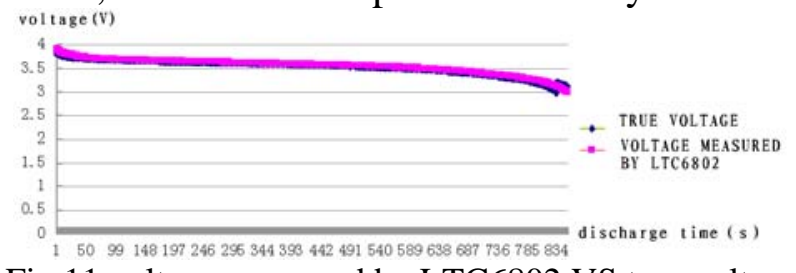

Fig.11 voltage measured by LTC6802 VS true voltage

\section{Conclusion}

Based on the analysis on the character of lithium-ion battery, this paper presents a monomer battery monitor module in the BMS. Real-time battery monomer voltage and temperature are measured by the LTC6802 device and the MC9S12DP256 microcontroller will handle immediately when an overvoltage or undervoltage happens. A thermal model of a monomer battery is built to decide the specific spots to place the thermistors or temperature sensors. The accuracy of the LTC6802 device is verified by the test, which shows it can do the measurement by rule and line.

\section{References}

[1] Huang Xue-jie. Li-ion Battery and its Key Materials[J].Materials China, 2010,(8):46-51.

[2] Dai Yong-nian, Yang Bin, Ma Wen-hui, The Development of Li-ion Battery and Light-EV[J]. Advanced Materials Industry,2006.(9):16-18.

[3] Michael Kultgen, Jon Munson. Battery stack monitor extends life of Li-Ion batteries in hybrid electric vehicles[J]. Linear Technology, 19(1):1-2(2009).

[4] Sun Bei,Wei Xuezhe, Design of voltage monitoring module of stacked lithium-ion cells in series[J].Applied Mechanics and Materials Vos.29-321(2010), 888-1893.

[5] Yu Yuan-bin, Min Hai-tao. Design of battery management system hardware circuit and bench testing verifying for li-ion batteries. Advanced Materials Research[J], Vol. 512-515(2012), p 1032-1036.

[6] Lu Chun. Research on thermal management of power battery pack on electrical vehicles at low temperature[D]. Beijing Institute of Technology(2011). 